\title{
A New Global-Local Approach to Optimize School Meals Delivery
}

\author{
Manoel S. L. Neto ${ }^{1}$, Marly G. F. Costa ${ }^{1}$, Cícero F. F. C. Filho ${ }^{1}$ \\ ${ }^{1}$ Centro de Pesquisa e Desenvolvimento de Tecnologia Eletrônica e da Informação - \\ Universidade Federal do Amazonas (CETELI/UFAM) \\ CEP 69077-000 - Manaus - AM - Brazil \\ \{mslneto, marlygfcosta, cffcfilho\}egmail.com
}

\begin{abstract}
This paper proposes a hybrid approach to solve the capacitated vehicle routing problem with time window constraints (CVRPTW). In order to evaluate such approach, we propose a case study with the primary goal of defining the delivery routes of school meals in the public school system of Manaus city (Amazonas - Brazil). The optimized solution minimizes the traveled distance of the delivery vehicle and takes into account the capacity and time window time constrains. The approach present in this paper is classified as global-local. On one hand, the metaheuristic simulated annealing, usingthe swap operator, is applied in the global search. On the other hand, $A^{*}$ search algorithm is applied in the local search, in order to refine all solutions presented by the global search. Importantly, the main difference between such approach and the remaining ones present in the literature is that all solutions from the global search are optimized in the local search. The experimental evaluation compares the results with another approach, which will be presented throughout the paper, by means of computation time and total traveled distance.
\end{abstract}

\section{Introduction}

The Municipal Department of Education of Manaus city (SEMED) presents a high logistic cost for school meal delivery. Such problem worsened in March $4^{\text {th }}$, 2013, when the city hall established a Child Care Food Program with SEMED, which provides an increase number of meals for the students during the day. In the past, each student had only one meal per day. At present, the program provides each child with up to seven meals per day, which would depend on the educational mode.

This delivery system plays an important role in the logistic planning, once involves the acquisition and maintenance of a fleet of vehicles, besides fuel costs. It is absolutely essential that customer demand can be efficiently met in order to reduce this cost. Recognizing the important role played by the transport sector, Dantzig and Ramser, in 1959, introduced the Vehicle Routing Problem (VRP), which contributed to reduce the logistics costs for companies and the society.

According to Dantzig and Ramser (1959) the problem fits properly in the area of combinatorial optimization and it can be expressed using the following terms: a set of dispersed customers, a centralized depot, and a vehicle fleet that picks up items from that depot and delivery it to customers. Thus, it is necessary to determine a route plan in order to minimize the total transport cost, or the total traveled distance. However, due to its multiple variables and the huge number of possibilities of combining them, such problem 
is considered as NP-Hard, non-deterministic polynomial-time hard problem. Therefore, there is no polynomial time algorithm to find optimal solutions.

This research is based on an urban area from Amazônia, the city of Manaus. In 2015, the population of Manaus was 2,057,709 inhabitants that classified it as the seventh most populous city in Brazil. According to Brazilian Institute of Geography and Statistics (2015), $99.49 \%$ of its inhabitants live in urban areas and $0.51 \%$ in rural areas.

In 2015, Brazilian Institute of Geography and Statistics (2015) showed that most of the population was in the north and east side of the city, where Cidade Nova is the most populous neighborhood with 121,135 inhabitants. Therefore, this research is based on the eastern and northern zones of Manaus city, once they have the highest concentration of people. These zones form a region named as the region IV.

Marques et al. (2013) presented the first work focused on routing of school meal delivery for the public educational system of Manaus city, treating the problem as a Capacitated Vehicle Routing Problem (CVRP). In order to obtain a solution, Marques et al. used a Geographic Information Systems (GIS) tool. At the time the work was done, the routes used to distribute the meals were empirically determined, and the driver was responsible for defining the best route. Particularly, the work was organized in three stages. Firstly, a database was structured; secondly, the authors employed a group analysis using the nearest neighbor technique; and finally, the routing was performed with the Network Analyst tool.

The purpose of this work is the development of a new methodology for optimizing the routes of a school meal delivery system in the aforementioned region IV. The main feature presented in this work is the association, in the optimization process, of a global search and a local search, in order to solve a capacitated vehicle routing problem with time window (CVRPTW).

In the simulated annealing method, a global search using a swap operator generates solutions formed by a sequence of nodes of interest. Particularly, similar to Pop and Chira (2014), we name such solutions as global routes. In the sequence, clusters are then formed, taking into account the constraints of time window and of vehicle capacity (whichever occurs first). The clusterization process uses the Euclidian distance between nodes of the global routes. Then, a local search using the $A^{*}$ search algorithm is performed within each cluster, in order to determine the real path between two schools. Indeed, local searches are performed on all the solutions presented by the global search. In the local search, we calculate the distance between the nodes taking into account the real distance between streets in a map, rather than Euclidean distance between nodes. In this phase, the clusters composition can change, because the real distances are higher than the Euclidian distances employed in the clusterization process.

In the literature, similar works also use simulated annealing [Lin et al., 2006, Lin et al., 2006a, Sakakibara et al., 2010] to solve the VRP problem, but, differently from the work presented here, they do not associate a local search for every global search.

This paper is organized as follows. Section 2 shows the data used and how it was obtained. Section 3 presents the problem of routing vehicles with time window. Section 4 explains the operation of the Simulated Annealing (SA) and of the A* search algorithm. Section 5 shows how the new global-local approach has been modeled. Section 6 shows how the comparative method has been modeled. Section 7 presents the results obtained by the new 
approach and a comparison with the comparative method. Finally, section 8 presents conclusions and contributions.

\section{Data}

The Municipal Department of Education of Manaus city (SEMED), provided the data used in this work. Importantly, this database comprises information related to logistic data of the schools that belong to region IV and contains geo-referenced data of Manaus city. The database expresses all coordinates in the Universal Transverse Mercator (UTM) coordinate system. In addition, we vectorize region IV, so that all 4,100-connection points between streets (secondary nodes) are marked. We insert the locations of the depot center and of the schools as new nodes (interest nodes). Furthermore, we catalogue all the 6,144 streets by name, location of the beginning and end, and size (i.e., the distance between its beginning and end). Figure 1(a) shows a map of the city of Manaus, highlighting this region, while Figure 1(b) shows the geo-referenced data.
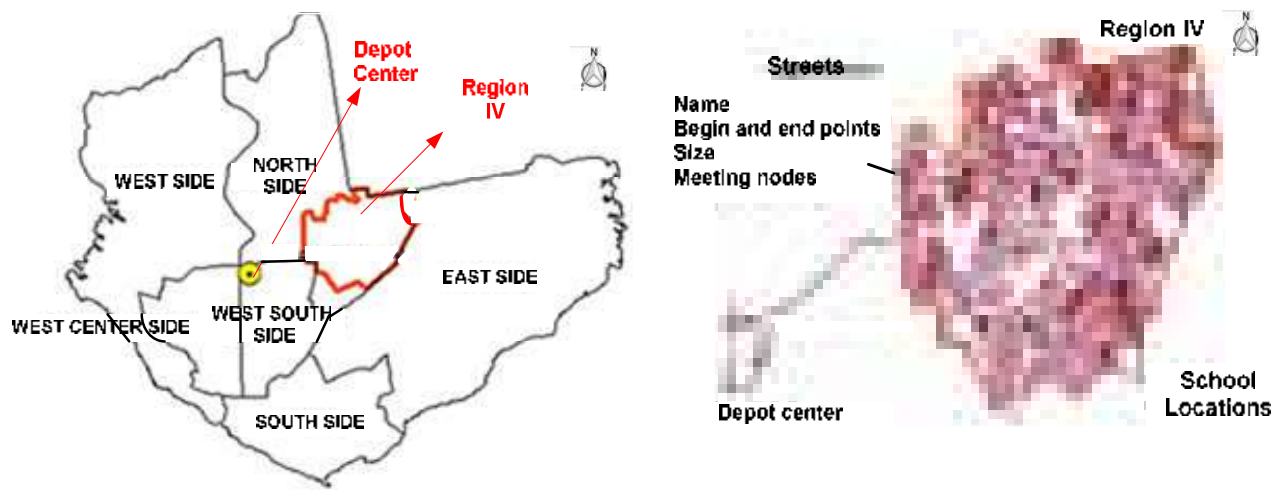

Figure 1: (a) Map of the city of Manaus showing Region IV, which is the focus of this research; (b) Georeferenced data of region IV

Meals are scheduled to be delivered between 8:00 a.m. until 5:30 p.m. The data from 53 schools is composed by: school name, modality, shifts, student capacity, and menus. Importantly, all data was collected directly from the logistics department of SEMED. The delivery vehicle for non-perishable foodstuffs is a truck (Tare: $6,590 \mathrm{~kg}$ | Net: 8,900 kg | Total gross: $14,990 \mathrm{~kg}$ ), which has a maximum permissible speed, in urban areas, of 60 $\mathrm{km} / \mathrm{h}$.

\section{Problem Description of the Capacitated Clustered Vehicle Routing Problem with Time Window}

A graph $G=(E, A)$, where $E=\{0, \ldots, n\}$ correspond to a set of vertices, defines a CCVRPTW. Traditionally, the number zero (0) represents the depot and the remaining ones, the served schools. Importantly, all routes start and finish at the depot, $E_{0}$. In addition, $A=\{(i, j) \mid i \in E, j \in E, i \neq j\}$ corresponds to a set of bows. In this context, the $\operatorname{cost} c_{i j}$ (distance between two vertices $i$ and $j$ ) and the traveled time $t_{i j}$ are associated to a respective bow $(i, j) \in A$. The symbol $t_{i j}$ represents the traveled time from school $i$ to school $j$, and includes the service time at school $i$. A set of identical vehicles is identified by $V=\{1, \ldots k\}$, where each vehicle has a capacity $Q$. Furthermore, each school $i \in E$ has 
a certain demand $d_{i}$ and, particularly, the depot demand $d_{0}$ is null. The vehicles must return to the depot during the time window $\left[a_{0}, b_{0}\right]$. In the problem just solved in this paper, $a_{0}=8: 00 \mathrm{am}$ and $b_{0}=5: 30 \mathrm{pm}$.

The model presented here has two decision variables. The former, $X_{i j}{ }^{k}$ which is defined to $\forall(i, j) \in E, \forall k \in V$, is equal to 1 if a vehicle $k$ follows the path from school $i$ to $j$, or 0 otherwise. The latter, $S_{i}{ }^{k}$, defined by $\forall i \in E, \forall k \in V$, represents the time that vehicle $k$ takes to reach the school $i$. If a certain vehicle $k$ does not perform any service in school $i$, then $S_{i}{ }^{k}$ is equal to zero. The sum of all demands $d_{i}$ from all schools served by a certain vehicle must be smaller than the respective vehicle capacity $Q$. The goal is to minimize the course route for each vehicle, such that all schools are served exactly once. All routes must be feasible according to the vehicles capacity and the time window $\left[a_{0}, b_{0}\right]$. One may note below a mathematical description of the problem:

Minimize

$$
\sum_{k \in V} \sum_{(i, j) \in A} c_{i j} X_{i j}^{k}
$$

Subject to

$$
\begin{gathered}
\sum_{k \in V} \sum_{j \in E} X_{i j}{ }^{k}=1, \forall i \in E \\
\sum_{i \in E} \sum_{j \in E}\left(d_{i}+d_{j}\right) X_{i j}{ }^{k} \leq Q, \forall k \in V \\
\sum_{j \in E} X_{0 j}{ }^{k}=1, \forall k \in V \\
\sum_{i \in E} X_{i h}{ }^{k}-\sum_{j \in E} X_{h j}{ }^{k}=0, \forall h \in E, \forall k \in V \\
\sum_{i \in E} X_{i, 0}{ }^{k}=1, \forall k \in V \\
X_{i j}{ }^{k}\left(S_{i}{ }^{k}+t_{i j}-S_{j}{ }^{k}\right) \leq 0, \forall(i, j) \in A, \forall k \in V \\
a_{0}+\sum_{i \in E} \sum_{j \in E} t_{i j} X_{i j}^{k}, \forall k \in V \leq b_{0} \\
X_{i j}{ }^{k} \in\{0,1\}, \forall(i, j) \in A, \forall k \in V
\end{gathered}
$$

Firstly, an objective function (1) denotes the minimization of the cost. The set of constrains (2) determines that each school must be assign to a respective vehicle. The set of constrains (3) determines that any vehicle cannot attend more schools than its capacity allow it. The sets of constrains (4), (5), and (6) correspond to flow constraints. Constraint (5) states that a vehicle $k$ that arrives at a school $h$ must depart from that school. Constraints (4) and (6) state that each vehicle $k$ leaves node 0 (depot) once and returns to node 0 . One may note that the set of constraints (6) is redundant, because constraint (5) encompasses it, but we hold it in the model in order to close the network structure. The set of constraints (7) denotes that a vehicle $k$ travels from $i$ to $j$ and, most important, it must not arrive at $j$ before the time $S_{i}^{k}+t_{i j}$ runs out. The set of constraints (8) ensures that all vehicles respect the time window. Finally, the set of constraints (9) is a set of integrality. 


\section{Global-Local Method}

As stated in the introduction, the proposed method comprises a global search undertaken by the SA algorithm and a local search undertaken by the $\mathrm{A}^{*}$ algorithm. Concerning the SA algorithm, we first perform a global search, employing the swap operator. In the sequence, clusters are then formed, taking into account the constraints of time window and of vehicle capacity (whichever occurs first). The clusterization process uses the Euclidian distance between nodes of the global routes. Then, a local search using the $A^{*}$ search algorithm is performed within each cluster, in order to determine the real path between two schools of region IV shown in Figure 1. In the local search, the clusters composition can change, because the real distances between nodes of interest are higher than the Euclidian distances employed in the clusterization process. The value of the objective function of the SA algorithm is the sum of the distances of the routes calculated by the local search. In the following section, we begin clarifying the two different problem representations that the method employs. One for the global search and the other for the local one.

\subsection{Solution Coding}

As stated, the proposed method uses a representation containing only the nodes of interests for generating neighbors in the SA algorithm and, for calculation of the objective function, employs other structure that incorporates the nodes that represent the intersections between the streets along the path from one school to another. For a better understanding, one can analyze the example shown in Figure 2, where D represents the depot node, E represents a school node, and $\mathrm{N}$ are nodes that represent crosses between streets. Each route start and finishes in the depot. Figure 2(a) shows two routes that start in the depot: route 1 is the following sequence of nodes D0-E1-E2-D0 and route 2, D0E3-E4-D0. In this paper, we name these routes as global routes. Figure 2(b) shows the same two routes of Figure 2(a), now including the crossing nodes $(\mathrm{N})$, that corresponds to the intersections of streets located in the path between two schools. Therefore, the local search route 1 is now represented by the following sequence of nodes D0-N2-N2-N1-N1N2-N3-N4-E2-N19-N20-D0 and route 2 by D0-N22-N12-N21-N13-E3-N8-N9-N10-E4N23-N24-N25-N26-N27-D0. This second way of route representation is used in the local search, by the $\mathrm{A}^{*}$ algorithm, for finding the best way between two schools. In this paper, we name these routes as local routes.

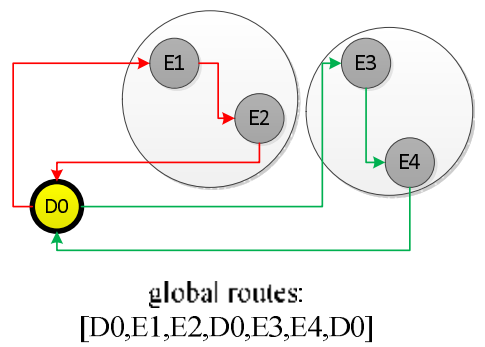

(a) 


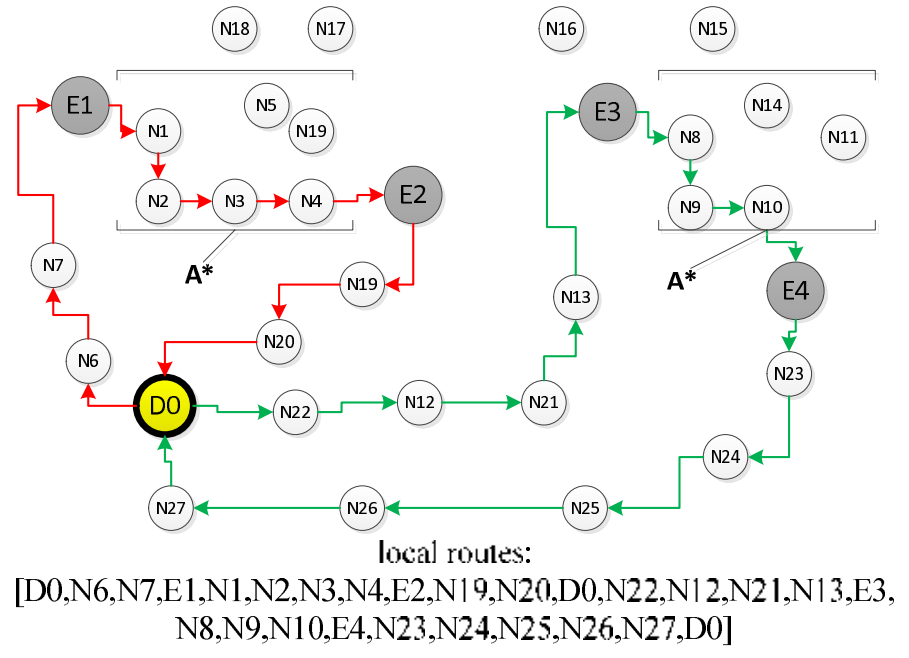

(b)

Figure 2. Problem representation (a) representation used for neighbor generation in the SA algorithm - global search; (b) representation used in the local search for finding the best way between two schools and for calculating the objective function of the SA algorithm.

\subsection{Simulated Annealing Algorithm}

Figure 3 shows the block diagram for the SA algorithm proposed to solve the CVRPTW problem. This SA algorithm is an adaptation of the original SA algorithm, and presents the following characteristics:

1. The first solution is random generated.

2. The SA algorithm comprises a global and a local search. For the neighbor generation step of the SA algorithm, we perform a global search, using the global route representation described earlier. For each global solution, local searches generate best paths between nodes of interests, using the local route representations described earlier.

3. The route (clusters) definitions happens through the time window or the limit of vehicle capacity, whichever occurs first. 


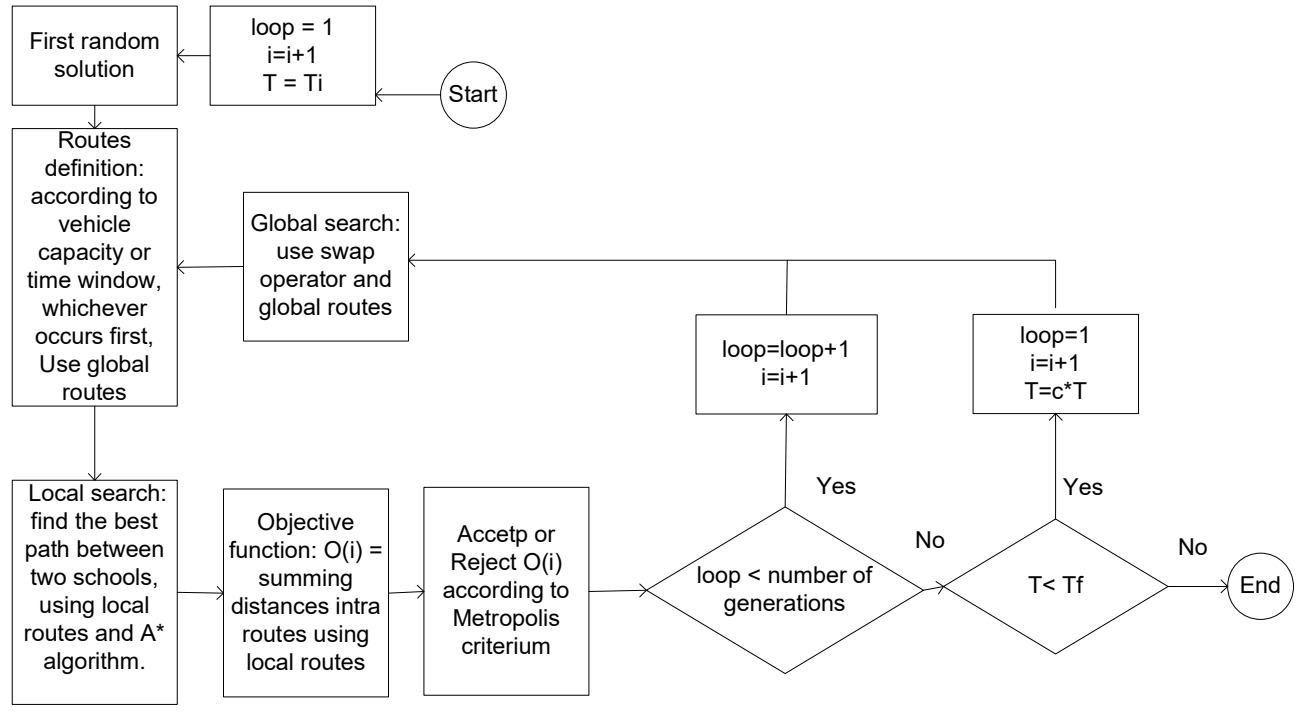

Figure 3: Block diagram of SA algorithm used in the global-local method

In order to perform the simulations, we use an Intel Core i7 with $3.60 \mathrm{GHz}$ and $16 \mathrm{~GB}$ of RAM, operating under the Microsoft Windows 8 platform. In addition, the program that simulates the planning of the delivery routes uses Python language.

\subsection{Objective function calculation}

The sum of intra-cluster distances, using local routes, results in the value of the objective function of the SA algorithm. For the local routes, given in section 4.1, we calculate the objective function according to the expressions (10), (11) and (12).

$$
\begin{gathered}
f_{\text {route } 1}=D_{D o \rightarrow N 6}+D_{N 6 \rightarrow N 7}+D_{N 7 \rightarrow E 1}+D_{E 1 \rightarrow N 1}+D_{N 1 \rightarrow N 2}+D_{N 2 \rightarrow N 3}+ \\
D_{N 3 \rightarrow N 4}+D_{N 4 \rightarrow E 2}+D_{E 2 \rightarrow N 19}+D_{N 19 \rightarrow N 20}+D_{N 20 \rightarrow D 0} \\
f_{\text {route } 2}=D_{D o \rightarrow N 22}+D_{N 22 \rightarrow N 12}+D_{N 12 \rightarrow N 21}+D_{N 21 \rightarrow N 13}+D_{N 13 \rightarrow E 3}+ \\
D_{E 3 \rightarrow N 8}+D_{N 8 \rightarrow N 9}+D_{N 9 \rightarrow N 10}+D_{N 10 \rightarrow E 4}+D_{E 4 \rightarrow N 23}+D_{N 23 \rightarrow N 24}+ \\
D_{N 24 \rightarrow N 25}+D_{N 25 \rightarrow N 26}+D_{N 26 \rightarrow N 27}+D_{N 27 \rightarrow D 0} \\
f_{\text {objective }}=f_{\text {route } 1}+f_{\text {route } 2}
\end{gathered}
$$

Where: $D_{A \rightarrow B}-$ Euclidean distance between nodes $A$ and $B$ of a local route.

\section{Comparative Method}

The new global-local method proposed in this paper, which was presented in section 4, will be compared with another similar approach, shown in Figure 4. 
In Figure 4 we show a block diagram of the SA algorithm used in the comparison method. This is a simpler method. The local search is employed only once, after the finishing of the internal and external loops. Different from the SA algorithm shown in Figure 3, the objetive function is calculated using distances among nodes of global routes. Considering the global routes shown in the example of Figure 2(a), the objetive function is calculated as shown in equations (13), (14) and (15).

$$
\begin{gathered}
f_{\text {route } 1}=D_{D o \rightarrow E 1}+D_{E 1 \rightarrow E 2}+D_{E 2 \rightarrow D 0} \\
f_{\text {route } 2}=D_{D o \rightarrow E 3}+D_{E 3 \rightarrow E 4}+D_{E 4 \rightarrow D 0} \\
f_{\text {objective }}=f_{\text {route } 1}+f_{\text {route } 2}
\end{gathered}
$$

Where: $D_{A \rightarrow B}-$ Euclidean distance between nodes $A$ and $B$ of a global route.

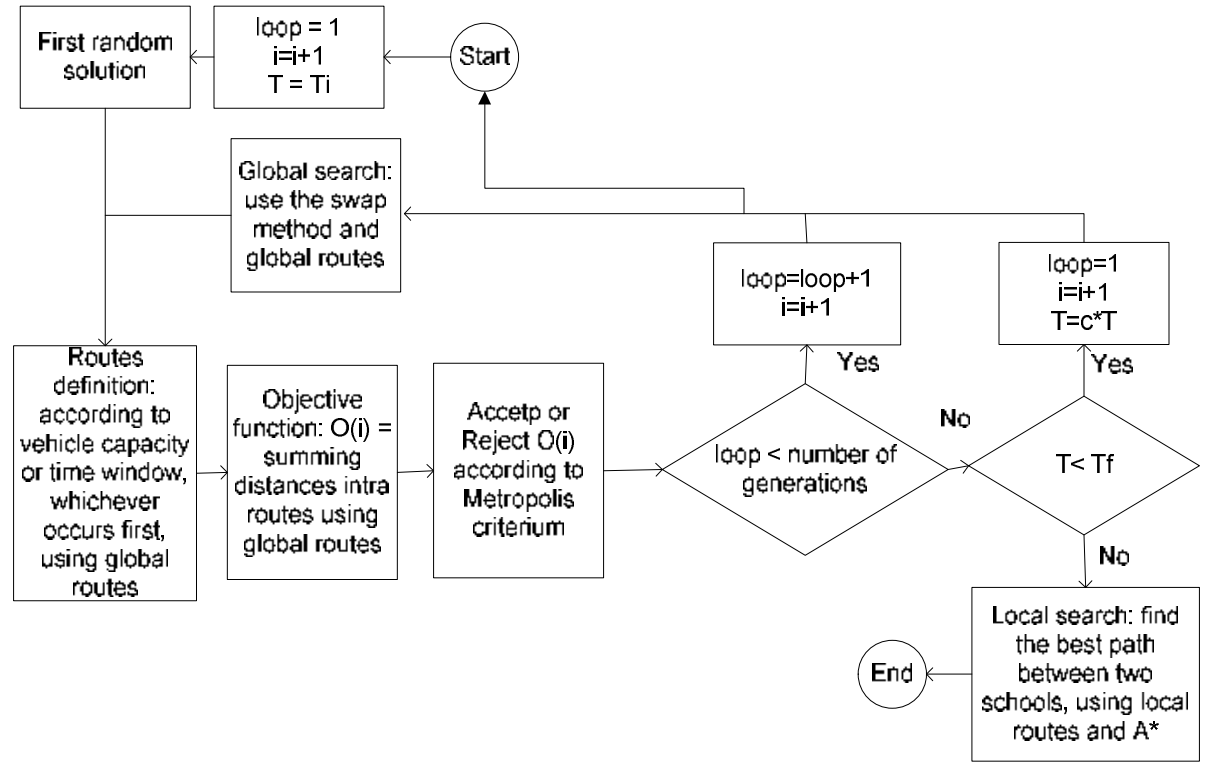

Figure 1. Comparative method

\section{Results}

In this section, we show the results obtained with the new global-local approach and the ones obtained with the comparative method. For the SA method, we evaluate the following initial temperatures: 200,160, 120, and 80. In addition, we evaluate the following number of generations: $5,10,15$, and 20 . Table 1 shows the obtained results. 
Table 1. Results obtained for the new global-local approach and for the comparative method.

\begin{tabular}{cccccc}
\hline \multirow{2}{*}{$\begin{array}{c}\text { Initial } \\
\text { Temperature }\end{array}$} & $\begin{array}{c}\text { Number of } \\
\text { generations }\end{array}$ & \multicolumn{2}{c}{ Propos ed method } & \multicolumn{2}{c}{ Comparative method } \\
\cline { 3 - 6 } & 5 & $\begin{array}{c}\text { Simulation } \\
\text { time }\end{array}$ & $\begin{array}{c}\text { Route } \\
\text { dis tance }\end{array}$ & $\begin{array}{c}\text { Simulation } \\
\text { time }\end{array}$ & $\begin{array}{c}\text { Route } \\
\text { distance }\end{array}$ \\
\hline \multirow{2}{*}{200} & 10 & $295,288.63$ & $683,311.61$ & 727.58 & $753,407.85$ \\
& 15 & $443,324.98$ & $661,031.25$ & 698.08 & $724,726.97$ \\
& 20 & $581,757.03$ & $621,775.61$ & 660.21 & $741,270.23$ \\
\hline \multirow{2}{*}{160} & 5 & $132,273.83$ & $711,489.18$ & $1,255.69$ & $858,179.35$ \\
& 10 & $268,664.75$ & $667,890.99$ & 885.25 & $803,470.34$ \\
& 15 & $387,218.38$ & $694,707.58$ & 972.84 & $740,822.64$ \\
& 20 & $542,059.34$ & $598,217.06$ & 556.80 & $742,836.33$ \\
\hline \multirow{2}{*}{120} & 5 & $123,674.5$ & $661,031.25$ & 974.00 & $795,632.89$ \\
& 10 & $255,368.82$ & $657,726.42$ & 675.95 & $712,833.53$ \\
& 15 & $373,032.78$ & $517,095.67$ & 774.00 & $750,340.77$ \\
& 20 & $498,744.89$ & $635,974.22$ & $1,095.80$ & $821,936.42$ \\
\hline \multirow{2}{*}{80} & 5 & $112,444.99$ & $598,217.06$ & $1,037.86$ & $711,489.18$ \\
& 10 & $242,663.73$ & $621,775.61$ & 827.03 & $850,038.30$ \\
& 15 & $341,334.55$ & $694,707.58$ & $1,114.15$ & $840,878.93$ \\
& 20 & $442,322.66$ & $618,316.45$ & 669.05 & $667,890.99$ \\
\hline
\end{tabular}

Table 1 shows that the proposed method presents higher simulation times than the comparative method. We believe that the main reason is that, in the comparative method, we employ the $A^{*}$ local search only once, after the end of the global search. In the proposed method, nevertheless, it is employed a number of times equal to the product between the number of generations in each temperature, the number of temperature values used in the simulated annealing cooling process and the number of clusters on each generation solution. For the route distance, nevertheless, the proposed method presents lower values than the comparative method. The main reasons for this behavior are the following: in the proposed method, we employ the local search for every interaction of the global search; in the proposed method, we calculate the objective function using the street distance, while in the global search of the comparative method, we calculate the objective function employing the Euclidean distance. The lower route distance found in the proposed method was 517,095.67 meters, while, in the comparative method, was $667,890.99$ meters.

\section{Conclusions}

The work presented in this paper proposes a new global-local approach to the resolution of the vehicle routing problem. This new approach was possible through the application of the simulated annealing metaheuristics, which is the same used in [Lin et al., 2006], [Lin et al., 2006a], and, [Sakakibara et al., 2010]. In addition, we employ the SA method to perform a global search using a swap operator. This neighborhood operator has previously been used in similar works, such as [Lin et al., 2006], [Mine et al., 2010], [Sakakibara et al., 2010], and [He et al., 2014]. Thereafter each global solution is found, the algorithm performs a division of the routes, based on the limitations of time and vehicle capacity. Then, within each route, a local search is performed by $A^{*}$ search 
algorithm. We carry on the calculation of the objective function using the real distance between streets in a map. Furthermore, the application of an exact algorithm using the distance between streets would be impracticable, due to the simulation times obtained.

We apply this new global-local approach to a case study of the Municipal Department of Education of Manaus city, more specifically in IV region, located between the north and east regions of Manaus, with 53 schools. The importance of such work can be gauged by the fact that, nowadays, in Manaus city, the local Department of Education is responsible for the delivery of school meals for more than 220,000 students in 500 schools. A decrease in the delivery time of these meals will have a huge impact on logistics costs.

As future work, we plan apply other methods to generate neighbors, whereas the swap method, as a random method, often initiate with schools far away from the depot. Furthermore, in order to reduce computational time, we plan to improve the response of the $\mathrm{A}^{*}$ search algorithm.

\section{References}

Assis, L. P., Maravilha, A. L., Vivas, A., Campelo, F. and Ramirez, J. A. (2013) "Multiobjective vehicle routing problem with fixed delivery and optional collections", Optimization Letters, v. 7, n. 7, p. 1419-1431.

Dantzig, G. B. and Ramser, J. H. (1959), “The Truck Dispatching Problem”, Management Science, v. 6, n. 1, p. 80-91.

Fanggeng, Z., Dong, M., Jiangsheng, S. and Weimin, L. (2009) "A hybrid genetic algorithm for the vehicle routing problem with simultaneous pickup and delivery", Chinese Control and Decision Conference, p.3928-3933.

Guan, C. H., Cao, Y. and Shi, J. (2010) "Tabu Search Algorithm for Solving the Vehicle Routing Problem", Third International Symposium on Information Processing, p.7477.

Hai, S., Yunlong, Z., Li, J. and Wenping, Z. (2010) "Two-phase heuristic for Capacitated Vehicle Routing Problem", Second World Congress on Nature and Biologically Inspired Computing (NaBIC), p.534-539.

He, Y., Miao, W., Xie, R. and Shi, Y. (2014) "A tabu search algorithm with variable cluster grouping for multi-depot vehicle routing problem", Proceedings of the 2014 IEEE 18th International Conference on Computer Supported Cooperative Work in Design (CSCWD), p.12-17.

Brazilian Institute of Geography and Statistics - IBGE (2015) “Arranjos Populacionais e Concentrações Urbanas do Brasil”, Geociências, D. D. e Geografia, C. D., Rio de Janeiro.

Lin, S. W., Ying, K. C., Lee, Z. J. and Chen, H. S. (2006) "Vehicle Routing Problems with Time Windows Using Simulated Annealing", IEEE International Conference on Systems, Man and Cybernetics, p.645-650.

Lin, S. W., Ying, K. C., Lee, Z. J. and Hsi, F. H. (2006) "Applying Simulated Annealing Approach for Capacitated Vehicle Routing Problems", IEEE International Conference on Systems, Man and Cybernetics, p.639-644. 
Marques, D. D. S., Araújo, C. S. D. and Rodrigues, H. F. (2013) "Roteirização do transporte da merenda escolar das escolas municipais urbanas de Manaus usando SIG", XX Simpósio de Engenharia de Produção - SIMPEP, Bauru, SP.

Mine, M. T., Silva, M. S. A., Ochi, L. S., Souza, M. J. F. and Silva, T. C. B. (2010) "O problema de roteamento de veículos com coleta e entrega simultânea: uma abordagem via Iterated Local Search e GENIUS”, Transportes, v. XVIII, n. 3, p. 60-71.

Pop, P. and Chira, C. (2014) "A hybrid approach based on genetic algorithms for solving the Clustered Vehicle Routing Problem", IEEE Congress on Evolutionary Computation (CEC), p.1421-1426.

Russell, S. J. and Norving, P. (2003) “Artificial intelligence: a modern approach”, $2^{\text {th }}$ edition.

Sakakibara, K., Tsuda, T. and Nishikawa, I. (2010) "Simulated annealing method based on recursive problem decomposition for vehicle routing problems", SICE Annual Conference, p.1016-1020. 